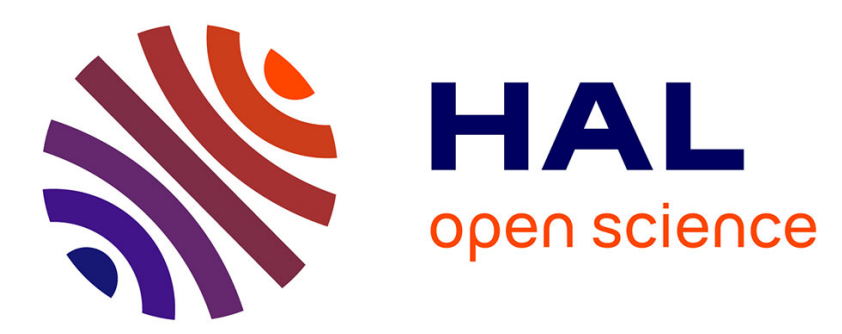

\title{
Triangulation sous contraintes en dimension quelconque
}

Houman Borouchaki

\section{To cite this version:}

Houman Borouchaki. Triangulation sous contraintes en dimension quelconque. [Rapport de recherche] RR-2373, INRIA. 1994. inria-00074303

\section{HAL Id: inria-00074303 https://hal.inria.fr/inria-00074303}

Submitted on 24 May 2006

HAL is a multi-disciplinary open access archive for the deposit and dissemination of scientific research documents, whether they are published or not. The documents may come from teaching and research institutions in France or abroad, or from public or private research centers.
L'archive ouverte pluridisciplinaire HAL, est destinée au dépôt et à la diffusion de documents scientifiques de niveau recherche, publiés ou non, émanant des établissements d'enseignement et de recherche français ou étrangers, des laboratoires publics ou privés. 
INSTITUT NATIONAL DE RECHERCHE EN INFORMATIQUE ET EN AUTOMATIQUE

\section{Triangulation sous contraintes en dimension quelconque}

Houman BOROUCHAKI

$\mathrm{N}^{\circ} 2373$

Octobre 1994

PROGRAMME 6

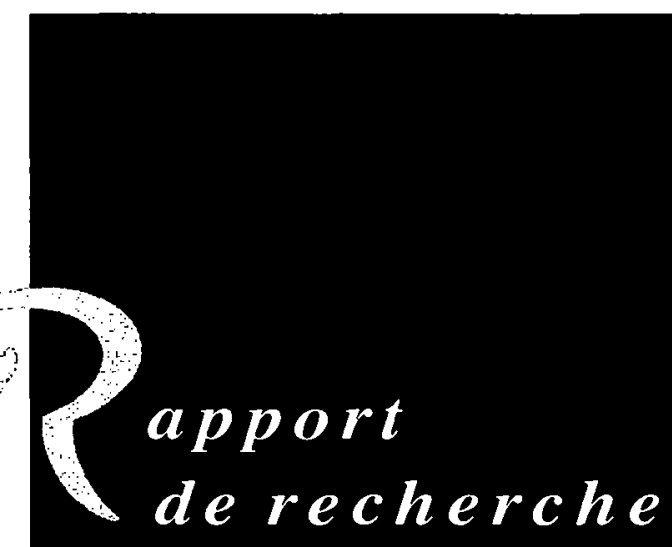


Les rapports de recherche de l'INRIA INRIA research reports sont disponibles en format postscript sous are available in postscript format ftp.inria.fr (192.93.2.54) ftp.inria.fr (192.93.2.54)

si vous n'avez pas d'accès ftp if you haven't access by ftp la forme papicr peut être commandée par mail : we recommend ordering them by e-mail : e-mail :dif.gesdif@inria.fr e-mail :dif.gesdif@inria.fr (n'oubliez pas de mentionner votre adresse postale). (don't forget to mention your postal address).

par courrier : by mail :

Centre de Diffusion Centre de Diffusion

INRIA INRIA

BP 105 - 78153 Le Chesnay Cedex (FRANCE) BP 105 - 78153 Le Chesnay Cedex (FRANCE) 


\title{
Triangulation sous contraintes en dimension quelconque
}

\author{
Houman BOROUCHAKI
}

INRIA, Domaine de Voluceau, Rocquencourt, BP 105, 78153 Le Chesnay Cedex, France.

\section{Résumé}

Il n'est pas toujours possible d'inclure une face de dimension quelconque dans une triangulation d'un nuage de points de $\mathbb{R}^{d}$ pour $d>2$, sans ajout de points de Steiner. Nous proposons une méthode simple qui consiste à inclure une triangulation de la face contrainte en ajoutant des points sur celle-ci. Ces points sont définis à partir des intersections de la face contrainte et des simplexes de la triangulation.

\section{Mots-clés}

Triangulation, Triangulation de Delaunay, Triangulation sous contraintes, Enveloppe convexe, Intersection de convexes.

\section{Constrained triangulation in any dimension}

\begin{abstract}
It is not always possible to insert a facet of any dimension in a triangulation of a finite set of points in $I R^{d}$ for $d>2$ without the adjunction of Steiner points. We propose here a simple method consisting in the inclusion of a triangulation of the constrained facet by adjunction of points upon it. These points are defined by using the intersections of the constrained facet with the simplices of the given triangulation.
\end{abstract}

\section{Key-Words}

Triangulation, Delaunay Triangulation, Constrained Triangulation, Convex Hull, Convex Intersection. 


\section{Introduction}

Les algorithmes de triangulation existent depuis longtemps. La plupart d'entre eux utilisent un graphe, dit d'adjacence, dont les noeuds sont les simplexes et les arêtes, les couples de simplexes adjacents. Parmi ces algorithmes, la triangulation de Delaunay, qui permet une manipulation aisée du graphe d'adjacence, occupe une place centrale. Elle impose des arêtes spéciales, dites de Dèlaunay, dans le graphe d'adjacence. Ces arêtes sont définies suivant le critère de la sphère vide [15]. Dans $\mathbb{R}^{2}$, on retrouve les arêtes de Delaunay en basculant des arêtes [21] dans le graphe d'adjacence. Ce n'est plus possible dans $\mathbb{I R}^{d}$, pour $d>2$ [24]; on peut cependant construire une certaine triangulation tout en effectuant des bascules d'arêtes [17][24][26] pour retrouver les arêtes de Delaunay.

On s'est intéressé au problème plus général : contraindre des arêtes quelconques dans un graphe d'adjacence donné c'est-à-dire inclure certaines $(d-1)$-faces dans une triangulation donnée. Ce problème est complètement résolu dans $\mathbb{R}^{2}[5][12][14][18][19][25][27][33]$, plus au moins résolu dans $\mathbb{R}^{3}$ [1][2][11][20][22][28][29][32], et restait ouvert dans $\mathbb{R}^{d}$ pour $d>3$. Pour $d \geq 3$, l'existence d'une solution n'est pas toujours garantie sans ajout de points de Steiner dans la triangulation. Le problème du nombre optimal de ces points est ouvert, même dans $I^{3}$.

Plusieurs algorithmes ont été proposés dans $\mathbb{R}^{3}$. Les uns partitionnent la face contrainte, à partir de points sur celle-ci. Les autres, en appliquant des bascules d'arêtes dans le graphe d'adjacence et en insérant des points non contenus dans la face si les bascules d'arêtes ne sont plus possibles, régénèrent la face contrainte. La seconde méthode a l'avantage de ne pas casser la face contrainte; mais comme la position des points supplémentaires insérés peut gêner l'insertion des autres faces contraintes, il faut connaître préalablement toutes les faces contraintes à insérer.

Nous présentons une méthode dans $\mathbb{I}^{d}$ pour l'insertion d'une face contrainte de dimension quelconque, en partitionnant cette face, nous inspirant des idées de [22] appliquées dans $\mathbb{R}^{3}$. Ce partitionnement est défini à partir des intersections de la face contrainte et des simplexes de la triangulation. On triangule les simplexes intersectés par la face contrainte, les uns indépendamment des autres, de telle sorte que les deux propriétés suivantes soient satisfaites :

a - la triangulation de chaque simplexe intersecté contient une triangulation du polytope résultant de l'intersection.

b - tout couple de simplexes intersectés adjacents induit la même triangulation sur leur $(d-1)$-face commune.

La première propriété assure la présence d'une partition de la face contrainte dans la triangulation et la seconde, une triangulation définie par l'ensemble des simplexes intersectés par la face contrainte. Ces deux propriétés seront réalisées grâce à̀ une triangulation particulière que l'on appelle simple.

Dans la deuxième section, on définit cette notion de triangulation simple et on établit des lemmes concernant les deux propriétés ci-dessus. La troisième section est un rappel sur la triangulation de Delaunay. L'algorithme de triangulation sous contraintes et quelques détails sur son implémentation font l'objet de la quatrième et de la cinquième section. Enfin on conclut sur l'énoncé de quelques sujets de recherche. 


\section{Triangulation Simple}

Soit $V$ un ensemble de $N$ points $\left(P_{i}\right)_{1 \leq i \leq N}$ de $I R^{d}$, donnés dans l'ordre lexicographique : $P_{i}<P_{j}$ si et seulement si

$$
\exists m, 1 \leq m \leq d, \quad \forall l<m \quad P_{i}^{l}=P_{j}^{l} \quad \text { et } \quad P_{i}^{m}<P_{j}^{m}
$$

où $P_{i}^{\prime}$ est la l-ième coordonnée de $P_{i}$.

Soit $V_{k}=\left\{P_{1}, \cdots, P_{k}\right\}$ l'ensemble des $k$ premiers points de $V$. Notons $\operatorname{Conv}\left(V_{k}\right)$ (resp. $\operatorname{Aff}\left(V_{k}\right)$ ), l'enveloppe convexe (resp. l'enveloppe affine) des points de $V_{k}$. Par définition des points de $V$, on a :

\section{LEMME 1. -}

Le point $P_{k+1}$ n'appartient pas à Conv $\left(V_{k}\right)$.

En effet $P_{k+1}>P_{1}$, il existe $m, 1 \leq m \leq d$, tel que $P_{k+1}^{m}>P_{1}^{m}$, et quel que soit $l, 1 \leq l<m$, $P_{k+1}^{l}=P_{1}^{l}$; comme quel que soit $\mathrm{j}, 1<j \leq k, P_{j}>P_{1}$, on a :

$$
\forall j, \quad 1<j \leq k, \quad \text { et } \quad \forall l, \quad 1<l<m, \quad P_{k+1}^{l}=P_{j}^{l}
$$

et

$$
\forall j, \quad 1<j \leq k, \quad P_{k+1}^{m} \geq P_{j}^{m} .
$$

Soient les réels $\left(\alpha_{j}\right)_{1 \leq j \leq k}$ vérifiant :

$$
\forall j, \quad 1<j \leq k, \quad \alpha_{j} \geq 0 \quad \text { et } \quad \sum_{1 \leq j \leq k} \alpha_{j}=1
$$

on a :

$$
\forall j, \quad 1<j \leq k, \quad \sum_{1 \leq j \leq k} \alpha_{j} P_{j}^{m}<\sum_{1 \leq j \leq k} \alpha_{j} P_{k+1}^{m}=P_{k+1}^{m} .
$$

Ainsi $P_{k+1} \notin \operatorname{Conv}\left(V_{k}\right)$.

DÉfinition 1. -

Une face frontalière $f$ de Conv $\left(V_{k}\right)$ est visible du point $P_{k+1}$ si et seulement si

$$
\operatorname{Conv}\left(P_{k+1}, f\right) \cap \operatorname{Conv}\left(V_{k}\right)=f
$$

où Conv $\left(P_{k+1}, f\right)$ est l'enveloppe convexe des sommets de $f$ et du point $P_{k+1}$.

Cela revient à dire qu'il existe un hyperplan support de $\operatorname{Conv}\left(V_{k}\right)$ qui passe par $f$ et qui sépare Conv $\left(V_{k}\right)$ du point $P_{k+1}$. Comme $P_{k+1} \notin \operatorname{Conv}\left(V_{k}\right)$, une telle face existe toujours. On désigne l'ensemble des faces de dimension maximale de $\operatorname{Conv}\left(V_{k}\right)$, visibles du point $P_{k+1}$, par $F_{k}$.

Nous allons définir par récurrence la triangulation simple $T(V)$ de $V$ dans $A f f(V)$. Une formulation plus générale est donnée dans [7].

Construction 1. -

(a) $-T\left(V_{1}\right)=P_{1}$ dans $P_{1}$.

(b) - Si $T\left(V_{k}\right)$ est la triangulation simple de $V_{k}$ dans Aff $\left(V_{k}\right)$, alors $T\left(V_{k+1}\right)$ dans Aff $\left(V_{k+1}\right)$ est définie par

$$
T\left(V_{k+1}\right)=\left\{\begin{array}{cl}
T\left(V_{k}\right) \cup \bigcup_{j \in F_{k}} \operatorname{Conv}\left(P_{k+1}, f\right) & \text { si } P_{k+1} \in A f f\left(V_{k}\right) \\
\bigcup_{s \in T\left(V_{k}\right)} \operatorname{Conv}\left(P_{k+1}, s\right) & \text { si } P_{k+1} \notin A f f\left(V_{k}\right)
\end{array}\right.
$$


Supposons que la dimension de $\operatorname{Aff}\left(V_{k}\right)$ soit égale à $\delta_{k}$. Si $P_{k+1} \in \operatorname{Aff}\left(V_{k}\right)$ alors $\operatorname{Conv}\left(P_{k+1}, f\right)$ est un simplexe de $\mathbb{R}^{\delta_{k}}$ et si $P_{k+1} \notin \operatorname{Aff}\left(V_{k}\right)$, $\operatorname{Conv}\left(P_{k+1}, s\right)$ est un simplexe de $\mathbb{R}^{\delta_{k}+1}$. Par construction, dans les deux cas, l'intersection de deux nouveaux simplexes (définis à l'étape $k+1)$ est soit vide, soit une face $g=\operatorname{Conv}\left(P_{k+1}, h\right)$, où $h$ est une $l$-face de $T\left(V_{k}\right)\left(1 \leq l \leq \delta_{k}-1\right)$. Ainsi $T\left(V_{k+1}\right)$ définit une triangulation simple de $V_{k+1}$ dans $\operatorname{Aff}\left(V_{k+1}\right)$.

Pour $P_{k+1} \in \operatorname{Aff}\left(V_{k}\right), \bigcup_{f \in F_{k}} \operatorname{Conv}\left(P_{k+1}, f\right)$ est la triangulation d'un polytope que l'on appelle cône de visibilité de $P_{k+1}$ par rapport à $\operatorname{Conv}\left(V_{k}\right)$. Dans ce cas, la triangulation $T\left(V_{k+1}\right)$ est la réunion des simplexes de $T\left(V_{k}\right)$ et des simplexes de la triangulation du cône de visibilité de $P_{k+1}$ par rapport à $\operatorname{Conv}\left(V_{k}\right)$. Par conséquent, à chaque étape de la construction, une modification minimale est apportée à la triangulation.

Cette construction utilise le fait que le dernier point à insérer est en dehors de l'enveloppe convexe des points déjà insérés. Pour cela on a supposé que les points sont ordonnés dans l'ordre lexicographique, bien que cette condition ne soit pas obligatoire. Une conséquence immédiate de cette définition est que la triangulation de l'étape $k$ est conservée à l'étape $k+1$.

Soient $\delta$ la dimension de $\operatorname{Aff}(V)$ et $P_{i_{1}}, \cdots, P_{i_{6}}, P_{i_{\sigma+1}}$ les $\delta+1$ premiers points affinement indépendants de $V$. Le lemme suivant permet une construction de la triangulation simple de $V$ en considérant uniquement des simplexes de $\mathbb{R}^{\delta}$.

\section{LEMME 2. -}

On a :

$$
T(V)=T\left(P_{i_{1}}, \cdots, P_{i_{\delta}}, P_{i_{6+1}}, V^{\prime}\right)
$$

où $V^{\prime}$ est l'ensemble des points de $V$ ordonné dans l'ordre lexicographique, privé des points $P_{i_{1}}, \cdots, P_{i_{b}}, P_{i_{\delta+1}}$.

La triangulation $T\left(P_{i_{1}}, \cdots, P_{i_{6}}, P_{i_{\delta+1}}, V^{\prime}\right)$ est bien définie; en effet aucun point de $V^{\prime}$ n'appartient à Conv $\left(P_{i_{1}}, \cdots, P_{i_{6}}, P_{i_{\delta+1}}\right)$, sinon il serait l'un des points $P_{i_{j}}(1 \leq j \leq \delta+1)$. On a $P_{i_{1}}=P_{1}$, $P_{i_{2}}=P_{2}$ et pour tout $j, 3 \leq j \leq \delta$

$$
T\left(P_{i_{1}}, P_{i_{2}}, \cdots, P_{i_{j}}, P_{i_{j}+1}, \cdots, P_{i_{j+1}}\right)=T\left(P_{i_{1}}, P_{i_{2}}, \cdots, P_{i_{j}}, P_{i_{j+1}}, P_{i_{j+1}}, \cdots, P_{i_{j+1}-1}\right) \text {; }
$$

comme $P_{i_{j+1}} \notin \operatorname{Aff}\left(P_{i_{1}}, P_{i_{2}}, \cdots, P_{i_{j}}, P_{i_{j}+1}, \cdots, P_{i_{j+1}-1}\right)$, par construction les deux triangulations sont identiques.

La triangulation $T\left(P_{i_{1}}, \cdots, P_{i_{\delta}}, P_{i_{\delta+1}}\right)$ définit un simplexe initial $s_{1}$ de $T(V)$ dans $\mathbb{R}^{\delta}$; chaque point de $V^{\prime}$ appartient à $\operatorname{Aff}\left(P_{i_{1}}, \cdots, P_{i_{\delta}}, P_{i_{b+1}}\right)$; la construction de $T(V)$ à partir de $s_{1}$ utilise uniquement des simplexes de $\mathbb{R}^{\delta}$.

Nous allons montrer par la suite comment on peut construire une triangulation d'un simplexe de dimension $d$, induisant une triangulation d'un polytope convexe donné inclus dans ce simplexe et de dimension strictement inférieure à $d$.

LEMME 3. -

Soient $\mathcal{Q}=\operatorname{Conv}\left(Q_{1}, \cdots, Q_{q}\right)$ un polytope convexe de sommets $Q_{1}, \cdots, Q_{q}$ ordonnés dans l'ordre lexicographique. Soient des points $R_{1}, \cdots, R_{r}$ vérifiant

$$
R_{1} \notin \operatorname{Conv}\left(Q_{1}, \cdots, Q_{q}\right)
$$

et

$$
\forall k, 1 \leq k \leq r-1 \quad R_{k+1} \notin \operatorname{Conv}\left(Q_{1}, \cdots, Q_{q}, R_{1}, \cdots, R_{k}\right),
$$

alors la triangulation $T\left(Q_{1}, \cdots, Q_{q}, R_{1}, \cdots, R_{r}\right)$ contient un ensemble de faces constituant une triangulation de $\mathcal{Q}$. 
En effet cet ensemble de faces est défini par $T\left(Q_{1}, \cdots, Q_{q}\right)$ et est inclus dans la triangulation $T\left(Q_{1}, \cdots, Q_{g}, R_{1}, \cdots, R_{r}\right)$ d'après sa construction.

\section{REMARQUE. -}

La triangulation $T\left(Q_{1}, \cdots, Q_{q}, R_{1}, \cdots, R_{r}\right)$ est bien définie bien que ces points ne soient pas ordonnés dans l'ordre lexicographique.

Comme cas particulier du lemme 3 on a :

LEMME 4. -

Si les points $\left(R_{i}\right)_{1 \leq i \leq d+1}$ sont les sommets d'un simplexe $s$ de dimension $d$ et si $s$ contient les points $\left(Q_{i}\right)_{1 \leq i \leq q}$ ordonnés dans l'ordre lexicographique, alors les points $\left(R_{i}\right)_{1 \leq i \leq d+1}$ vérifient les propriétés du lemme 3 et la triangulation

$$
\mathcal{T}_{s}=T\left(Q_{1}, \cdots, Q_{q}, R_{1}, \cdots, R_{d+1}\right)
$$

de $s$ contient un ensemble de faces constituant une triangulation de $\mathcal{Q}$.

L'ordre lexicographique pour les points $\left(R_{i}\right)_{1 \leq i \leq d+1}$ n'est pas nécessaire, mais il le devient pour garantir une triangulation unique de chaque $(d-1)$-face du simplexe $s$, indépendamment de la triangulation de $s$.

Notation.

Soit $G$ un ensemble de points. On note $\operatorname{Lex}(G)$ la suite des points de $G$ ordonnés dans l'ordre lexicographique.

\section{LEMME 5. -}

Soient $s_{\alpha}=\operatorname{Conv}\left(R_{\alpha}, g\right)$ et $s_{\beta}=\operatorname{Conv}\left(R_{\beta}, g\right)$ deux simplexes adjacents ayant la $(d-1)$ face commune $g=\operatorname{Conv}\left(R_{1}, \cdots, R_{d}\right)$. Soit $G$ la réunion de trois ensembles disjoints $G_{\alpha}=$ $\left\{\left(Q_{\alpha_{1}}\right)_{1 \leq i \leq r_{\alpha}}\right\}$ contenu dans $s_{\alpha}-g, G_{\beta}=\left\{\left(Q_{\beta_{0}}\right)_{1 \leq i \leq r_{\beta}}\right\}$ contenu dans $s_{\beta}-g$ et $G_{\gamma}=\left\{\left(Q_{\gamma_{i}}\right)_{1 \leq i \leq r_{\gamma}}\right\}$ contenu dans $g$. Alors les deux triangulations

$$
\mathcal{T}_{s_{\alpha}}=T\left(\operatorname{Lex}\left(G_{\alpha} \cup G_{\gamma}\right), \operatorname{Lex}\left(\left\{R_{1}, \cdots, R_{d}, R_{\alpha}\right\}\right)\right)
$$

et

$$
\mathcal{T}_{s_{\beta}}=T\left(\operatorname{Lex}\left(G_{\beta} \cup G_{\gamma}\right), \operatorname{Lex}\left(\left\{R_{1}, \cdots, R_{d}, R_{\beta}\right\}\right)\right)
$$

induisent la même triangulation sur $g$.

En effet, d'après le lemme $4, \mathcal{T}_{s_{\alpha}}$ (resp. $\mathcal{T}_{s_{\beta}}$ ) est une triangulation de $s_{\alpha}$ (resp. $s_{\beta}$ ) bien définie. Dans ces deux cas, la triangulation induite sur $g$ est définie par

$$
T\left(\operatorname{Lex}\left(G_{\gamma}\right), \operatorname{Lex}\left(\left\{R_{1}, \cdots, R_{d}\right)\right),\right.
$$

car les deux points $R_{\alpha}, R_{\beta}$ et les points des deux ensembles $G_{\alpha}$ et $G_{\beta}$ n'interviennent pas dans la triangulation de $g$.

REMARQUE. -

Supposons $G_{\gamma} \cap\left\{R_{1}, \cdots, R_{d}\right\} \neq \emptyset$. Soient $R_{i_{1}}, \cdots, R_{i_{r}}$ les sommets de $g$ non contenus dans $G_{\gamma}$, alors on a :

$$
\mathcal{T}_{s_{\alpha}}=T\left(\operatorname{Lex}\left(G_{\alpha} \cup G_{\gamma}\right), \operatorname{Lex}\left(\left\{R_{i_{1}}, \cdots, R_{i_{r}}, R_{\alpha}\right\}\right)\right)
$$

et

$$
\mathcal{T}_{s_{\beta}}=T\left(\operatorname{Lex}\left(G_{\beta} \cup G_{\gamma}\right), \operatorname{Lex}\left(\left\{R_{i_{1}}, \cdots, R_{i_{r}}, R_{\beta}\right\}\right)\right)
$$

et la triangulation de $g$ est définie par

$$
T\left(\operatorname{Lex}\left(G_{\gamma}\right), \operatorname{Lex}\left(\left\{R_{i_{1}}, \cdots, R_{i_{r}}\right)\right) .\right.
$$




\section{Triangulation de Delaunay}

La triangulation de Delaunay d'un nuage de points est une triangulation à sphères vides. En d'autres termes les boules ouvertes circonscrites aux simplexes de la triangulation ne contiennent aucun point du nuage.

A cause de ses nombreuses applications dans différents domaines de la science, cette triangulation est devenue très populaire. Au départ elle représentait le dual des polytopes de Voronoï associés [15][35]. Sa régularité dans $\mathbb{R}^{2}$ est immédiate alors que sa régularité dans $\mathbb{I}^{d}$ pour $d>2$ est une conjecture. Dans [31], on démontre son optimalité en un certain sens.

Nous utilisons cette triangulation comme point de départ de notre algorithme de triangulation sous contraintes, bien qu'une triangulation quelconque puisse constituer la triangulation initiale des points de $V$.

Divers algorithmes [3][4][7][8][16][17][23][31][36] ont été proposés pour la construction de la triangulation de Delaunay d'un nuage de points dans $\mathbb{R}^{d}$. Nous retenons celui de [7], que nous rappelons sans démonstration.

On suppose toujours les points de $V$ ordonnés dans l'ordre lexicographique et on va définir, par récurrence, la triangulation de Delaunay $T^{d}(V)$ de $V$ dans $\operatorname{Aff}(V)$. La construction est définie en deux étapes; la première est similaire à celle d'une triangulation simple et la seconde est une correction dite de Delaunay.

Construction 2. -

(a) $-T^{d}\left(V_{1}\right)=P_{1}$ dans $P_{1}$.

(b) - Si $T^{d}\left(V_{k}\right)$ est la triangulation de Delaunay de $V_{k}$ dans $A f f\left(V_{k}\right)$, alors $T^{d}\left(V_{k+1}\right)$ dans Aff( $\left(V_{k+1}\right)$ est définie par

$$
\begin{gathered}
T^{\prime d}\left(V_{k+1}\right)=T^{d}\left(V_{k}\right) \cup \bigcup_{J \in F_{k}} \operatorname{Conv}\left(P_{k+1}, f\right) \quad \text { si } P_{k+1} \in A \text { ff }\left(V_{k}\right) \\
T^{d}\left(V_{k+1}\right)=\left\{\begin{array}{cl}
\left(T^{\prime d}\left(V_{k+1}\right) \backslash \mathcal{E}_{k}\right) \cup \bigcup_{f \in E_{k}} \operatorname{Conv}\left(P_{k+1}, f\right) & \text { si } P_{k+1} \in A f f\left(V_{k}\right) \\
\bigcup_{s \in T^{d}\left(V_{k}\right)} \operatorname{Conv}\left(P_{k+1}, s\right) & \text { si } P_{k+1} \notin A f f\left(V_{k}\right)
\end{array}\right.
\end{gathered}
$$

où $\mathcal{E}_{k}$ est l'ensemble des simplexes dont l'un des sommets est $P_{k+1}$ ou dont la boule circonscrite contient $P_{k+1}$ et $E_{k}$ est l'ensemble des faces frontalières de $\mathcal{E}_{k}$ qui ne contiennent pas le point $P_{k+1}$.

\section{Triangulation sous contraintes}

Soit $f=\operatorname{Conv}\left(P_{i_{1}}, \cdots, P_{i_{r}}\right)$ une face de dimension $r-1$, dont les sommets appartiennent à $V$. On considère le problème suivant :

PROBLÈME 1. -

Construire une triangulation $T_{f}(V)$ de $V$ telle que $f$ soit une face d'un simplexe de $T_{f}(V)$.

Pour $d>2$, il n'y a pas toujours de solution sans insertion de points de Steiner [32]. Pour $d=$ 3 ce problème est NP-complet [32]. Nous proposons une solution approximative en considérant une variante du problème 1 : 


\section{Problème 2. -}

Construire une triangulation $T_{f}\left(V \cup V_{f}\right)$ de $V \cup V_{f}$ telle qu'un ensemble de faces des simplexes de cette triangulation constitue une triangulation de $f$, où $V_{f}$ est un ensemble de points de $f$, ̀̀ déterminer.

La triangulation de $f$ est alors définie à partir de la réunion de ses sommets et de $V_{f}$. En d'autres termes on propose de "casser" la face pour l'inclure dans la triangulation.

\section{Algorithme DE TRIANGULATION SOUS CONTRAintes. -}

On considère d'abord une triangulation initiale de $V$, en particulier la triangulation de Delaunay $T^{d}(V)$ de $V$. Puis on recherche les simplexes intersectés par la face contrainte $f$; on triangule chacun de ces simplexes de telle sorte que son intersection avec $f$ soit une réunion de faces et que la triangulation induite sur ses $(d-1)$-faces soit unique. Finalement il reste à établir les relations d'adjacence entre les nouveaux simplexes.

L'algorithme est décrit par le schéma suivant :

\begin{tabular}{|c|}
\hline Triangulation de Delaunay $T^{d}(V)$ de $V$ \\
\hline Recherche des simplexes intersectés par $f$ \\
\hline Triangulation sous contrainte de chaque simplexe intersecté par $f$ \\
\hline Etablissement des nouvelles relations d'adjacence \\
\hline
\end{tabular}

Soient $S_{f}$ l'ensemble des simplexes intersectés par la face contrainte $f$ et $s$ un élément quelconque de $S_{f}$. On a :

$$
s \cap f=\operatorname{Conv}\left(Q_{s, 1}, \cdots, Q_{s, q_{s}}\right)
$$

où $\operatorname{Conv}\left(Q_{s, 1}, \cdots, Q_{s, q_{s}}\right)$ est un polytope convexe de dimension au plus égale à la dimension de $f$, dont les sommets sont les points $\left(Q_{s, i}\right)_{1 \leq i \leq q}$.

\section{LEMME 6. -}

Les points $\left(Q_{s, i}\right)_{1 \leq i \leq q}$, appartiennent aux $(d-1)$-faces de $s$. Si un sommet de $s$ appartient $\dot{a} s \cap f$ alors ce sommet est l'un des points $\left(Q_{s, i}\right)_{1 \leq i \leq q}$. vide.

En effet aucun sommet de $f$ n'est intérieur à $s$; car tout simplexe de $T(V)$ a un intérieur

Supposons $s=\operatorname{Conv}\left(R_{s, 1}, \cdots, R_{s, d+1}\right)$. Définissons la triangulation $\mathcal{T}_{s}$ de $s$ par la construction suivante :

Construction 3 . -

Si aucun sommet de $s$ n'appartient à $s \cap f$ alors

$$
\mathcal{T}_{s}=T\left(\operatorname{Lex}\left(\left\{Q_{s, 1}, \cdots, Q_{s, q,}\right\}\right), \operatorname{Lex}\left(\left\{R_{s, 1}, \cdots, R_{s, d+1}\right\}\right)\right)
$$

sinon

$$
T_{s}=T\left(\operatorname{Lex}\left(\left\{Q_{s, 1}, \cdots, Q_{s, q_{s}}\right\}\right), \operatorname{Lex}\left(\left\{R_{s, i_{1}}, \cdots, R_{s, i_{r}}\right\}\right)\right)
$$

où $R_{s, i_{1}}, \cdots, R_{s, i_{r}}$ sont les sommets de $s$ non contenus dans $f$.

D'après le lemme $4, \mathcal{T}_{s}$ est bien définie et respecte les contraintes constituant une triangulation de $s \cap f=\operatorname{Conv}\left(Q_{s, 1}, \cdots, Q_{s, q_{s}}\right)$. 


\section{REMARQUE. -}

S'il existe des sommets de $s$ appartenant à $s \cap f$, on les insère, dans la triangulation $\mathcal{T}_{s}$, une seule fois en tant que sommets de $\operatorname{Conv}\left(Q_{s, 1}, \cdots, Q_{s, q_{s}}\right)$ et non en tant que sommets de $s$.

THÉORÈME 1. -

La réunion $\bigcup_{s \in S_{f}} \mathcal{T}_{s}$ définit une triangulation de

$$
\bigcup_{s \in S_{f}}\left\{R_{s, 1}, \cdots, R_{s, d+1}\right\} \cup \bigcup_{s \in S_{f}}\left\{Q_{s, 1}, \cdots, Q_{s, q_{s}}\right\}
$$

où les points $R_{s, 1}, \cdots, R_{s, d+1}$ sont les sommets de

$$
s=\operatorname{Conv}\left(R_{s, 1}, \cdots, R_{s, d+1}\right)
$$

et les points $\left(Q_{s, i}\right)_{1 \leq i \leq q}$, sont les sommets de

$$
s \cap f=\operatorname{Con} v\left(Q_{s, 1}, \cdots, Q_{s, q_{s}}\right) .
$$

Il suffit de vérifier, pour chaque couple de simplexes adjacents de $S_{f}$, que l'on a la même triangulation induite sur leur $(d-1)$-face commune. Soient $\left(s_{\alpha}, s_{\beta}\right)$ un tel couple, $g$ leur $(d-1)$ face commune et $G$ l'ensemble $\left\{Q_{s_{\alpha}, 1}, \cdots, Q_{s_{\alpha}, q_{\alpha_{\alpha}}}, Q_{s_{\beta}, 1}, \cdots, Q_{s_{\beta}, q_{\circ},}\right\}$. En posant $G_{\gamma}=G \cap g$, $G_{\alpha}=G \cap\left(s_{\alpha}-g\right)$ et $G_{\beta}=G \cap\left(s_{\beta}-g\right)$, on est dans la situation du lemme 5 et les deux triangulations $\mathcal{T}_{s_{\alpha}}$ et $\mathcal{T}_{s,}$ induisent sur $g$ la même triangulation.

La triangulation $\bigcup_{s \in S_{f}} \mathcal{T}_{\text {s }}$ respecte les contraintes constituées par

$$
\bigcup_{s \in S,} T\left(\operatorname{Lex}\left(\left\{Q_{s, 1}, \cdots, Q_{s, q,}\right\}\right)\right)
$$

définissant une triangulation de la face contrainte $f$.

Pour chaque simplexe triangulé, les relations d'adjacence des simplexes constituant la triangulation sont déjà determinées; il reste à établir les relations existant entre tous les couples de simplexes adjacents triangulés. Pour un couple fixé, il suffit d'établir la relation d'adjacence pour une paire de simplexes (dont l'un appartient à la triangulation du premier simplexe du couple et l'autre à la triangulation du seconde simplexe du couple) qui contiennent une $(d-1)$-face appartenant à la $(d-1)$-face commune des simplexes du couple, puis d'utiliser des techniques décrites dans [7] pour l'établissement des relations d'adjacence des autres simplexes contenant une $(d-1)$-face qui appartient à la $(d-1)$-face commune des simplexes du couple.

\section{Implémentation}

Nous rappelons qu'une triangulation est définie par un graphe dit de connexion [7] dont les noeuds sont les simplexes et les arêtes les couples de simplexes adjacents. A chaque noeud est associé une matrice dite de connexion qui permet de repérer l'indexage des sommets des noeuds voisins. Ceci facilite le parcours, à travers la triangulation, simplexe par simplexe, conservant une $(d-2)$-face.

D'après le schéma de construction d'une triangulation sous contrainte, trois outils sont indispenșables :

a - la construction incrémentale d'une triangulation simple. 
b - la construction incrémentale d'une triangulation de Delaunay.

c - le calcul des sommets du polytope convexe résultant de l'intersection d'une face et d'un simplexe.

Les algorithmes de construction incrémentale d'une triangulation simple et d'une triangulation de Delaunay, qui sont entre autres des mises à jour du graphe de connexion associé à la triangulation, sont données dans [7]. I nous reste à décrire l'algorithme d'intersection d'une face et d'un simplexe.

Dans $\mathbb{R}^{2}$ (resp. $I R^{3}$ ), le problème se ramène au calcul d'intersection de deux segments (resp. un segment et un triangle). En effet, dans ces cas, les sommets recherchés appartiennent aux arêtes de la face ou du simplexe. Cette propriété n'est plus valable en dimension $d>3$. Pour $d>3$, on propose une solution en transformant le problème d'intersection en un problème d'enveloppe convexe d'un nuage de points pour lequel des algorithmes de construction sont proposés dans [6].

Soit $g$ une $(d-1)$-face et $H_{g}$ l'hyperplan passant par $g$. Supposons que $O \notin H_{g}$, où $O$ est l'origine des coordonnées. L'équation du demi-espace défini par $H_{g}$ et contenant $O$, peut s'écrire sous la forme

$$
\sum_{1 \leq i \leq d} n_{H_{9}, i} x_{i}<1
$$

où $\left(n_{H_{g}, i}\right)_{1 \leq i \leq d}$ est un vecteur normal de $H_{g}$.

On obtient alors une dualité, que nous désignons par $\delta$, entre les hyperplans $H$ vérifiant $O \notin H$ et les points $\delta(H)=\left(n_{H, i}\right)_{1 \leq i \leq d}$ de $\mathbb{R}^{d}$ vérifiant

$$
\forall x=\left(x_{i}\right)_{1 \leq i \leq d} \in H \quad \sum_{1 \leq i \leq d} n_{H, i} x_{i}=1 .
$$

Inversement, à chaque point $P \neq O$ de $\mathbb{R}^{d}$, on associe l'hyperplan défini par l'équation

$$
\delta(P)=\sum_{1 \leq i \leq d} P^{i} x_{i}=1
$$

où $P^{i}$ est la i-ième coordonnée du point $P$.

Ràppelons quelques résultats concernant cette dualité [30] :

LEMME 7. -

Soient $s$ un simplexe contenant strictement l'origine, $f_{1}, \cdots, f_{d+1}$ ses $(d-1)$-faces. Soit $P$ un point interieur à $s$. Alors

$$
\delta(P) \cap \operatorname{Conv}\left(\delta\left(H_{f_{1}}\right), \cdots, \delta\left(H_{f_{d+1}}\right)\right)=\emptyset
$$

où $H_{f_{j}}$ pour $1 \leq j \leq d$ est l'hyperplan qui passe par la $(d-1)$-face $f_{j}$.

L'hyperplan $\delta(P)$ est défini par $\delta(P)=\sum_{1 \leq i \leq d} P^{i} x_{i}=1$. Comme $P$ est intérieur à $S$, on a :

$$
\forall j 1 \leq j \leq d+1 \quad \sum_{1 \leq i \leq d} n_{f_{j, i}} P^{i}<1
$$

Ces relations montrent que les points $\left(n_{f_{j, i}}\right)_{1 \leq i \leq d}(1 \leq j \leq d+1)$ et l'origine $O$ appartiennent tous au demi-espace défini par $\sum_{1 \leq i \leq d} P^{i} x_{i}<1$. Par conséquent l'intersection de $\delta(P)$ et de l'enveloppe convexe de ces points est vide.

Remarquons que $O$ est intérieur à $\mathcal{C}=\operatorname{Conv}\left(\delta\left(H_{f_{1}}\right), \cdots, \delta\left(H_{f_{d+1}}\right)\right)$; sinon il existerait un hyperplan séparant $O$ des points $\delta\left(H_{f_{j}}\right)(1 \leq j \leq d)$, ce qui est impossible. Chaque point $\delta\left(H_{f_{j}}\right)$ 
pour $1 \leq j \leq d+1$ est un sommet $\operatorname{de} \mathcal{C}$; sinon il lui serait interne et d'après le lemme 7 (généralisé au cas des simplexes de dimension quelconque) son dual, l'hyperplan $H_{f_{j}}$, n'intersecterait pas $s$. On en déduit que le dual $\delta(s)$ de $s$ est un simplexe contenant l'origine $O$. Une conséquence immédiate est le lemme suivant :

\section{LEMME 8. -}

Soient s (resp. $t$ ) un simplexe contenant strictement l'origine, $f_{1}, \cdots, f_{d+1}$ (resp. $\left.g_{1}, \cdots, g_{d+1}\right)$ ses $(d-1)$-faces. On a :

$$
\delta(s \cap t)=\operatorname{Conv}\left(\delta\left(H_{f_{1}}\right), \cdots, \delta\left(H_{f_{d+1}}\right), \delta\left(H_{g_{1}}\right), \cdots, \delta\left(H_{g_{d+1}}\right)\right)
$$

En d'autres termes l'intersection de deux simplexes contenant l'origine est le dual de l'enveloppe convexe des points duaux des hyperplans passant par les $(d-1)$-faces de ces simplexes. Chaque sommet du polytope résultant de l'intersection est alors le dual d'un hyperplan contenant une $(d-1)$-face frontalière de cette enveloppe convexe.

Considérons le problème d'intersection d'une face $f$ et d'un simplexe $s$. On suppose, dans un premier temps, que $f$ est de dimension $d-1$. Soit $X$ un point interne de $s$ non contenu dans $f$ : par exemple l'un des points $\left(W_{i}\right)_{1 \leq i \leq d+1}$, non contenu dans $f$, dont toutes les coordonnées barycentriques sont égales à $\frac{1}{2(d+1)}$ sauf la i-ième qui est égale à $\frac{d+2}{2(d+1)}$. Soit le point $Y$ défini en sorte que $X$ soit le barycentre du simplexe $t=\operatorname{Conv}(Y, f)$. Les simplexes $s$ et $t$ contiennent dans leur intérieur le point $X$. En faisant un changement de repère pour que $X$ soit l'origine et en appliquant le lemme 8 , on trouve les sommets du polytope résultant de l'intersection de $s$ et de $t$. Parmi ces sommets on considère ceux qui appartiennent à $f$. Ces derniers sont les sommets de $s \cap f$. Si la face $f$ est de dimension strictement inférieure à $d-1$, on la complète en une $(d-1)$-face avec les sommets de $s$ non contenus dans l'enveloppe affine des sommets de $f$. Puis on applique le procédé précédent.

\section{Conclusion}

Nous avons proposé un algorithme d'insertion de face dans une triangulation quelconque, qui consiste à inclure une partition de la face dans la triangulation. Cette partition est définie à partir des intersections des simplexes de la triangulation et de la face contrainte. Nous avons supposé la face contrainte simpliciale; mais une généralisation au cas des faces non simpliciales est immédiate. In suffit de généraliser les algorithmes d'intersection des simplexes au cas des poly tope convexes.

La méthode proposée résout en partie le problème de la triangulation sous contrainte dans toute sa généralité. Toutefois elle ne permet pas d'imposer une arête quelconque dans un graphe d'adjacence donné. Une recherche future consiste à considérer les points, définissant la partition de la face contrainte, comme libres, à les déplacer à-travers la triangulation et retrouver la face contrainte. Ceci par exemple en utilisant des techniques d'optimisation de maillage décrits dans $[9]$.

Les simplexes intersectés par la face contrainte sont triangulés indépendamment. Ceci permet d'envisager des algorithmes parallèles: Le nombre de processeurs doit être proportionnel au nombre de simplexes de la triangulation initiale. Comme pour chaque simplexe intersecté par la face contrainte le nombre de simplexes constituant sa triangulation dépend de la dimension $d$, et non du nombre de points initiaux, alors le coût d'insertion d'une face est constant.

Encore une fois, le problème de recherche de l'enveloppe convexe d'un nuage de points est à la base de la méthode présentée. En effet les constructions incrémentales des triangulations 
simples et de Delaunay et la recherche de l'intersection d'une face et d'un simplexe se ramènent à une construction d'enveloppe convexe d'un nuage de points. Divers techniques de construction en dimension $d$ ont été proposé. Bien que des algorithmes optimaux de construction existent $[4][6][10][13][34]$, ce problème suscite toujours un grand interêt, notamment pour la recherche d'un algorithme parallèle en dimension $d$.

Une implémentation numérique des résultats décrits dans cet article est en cours de développement, ainsi que des applications aux problèmes d'interpolation et d'évolution posés dans $\mathbb{R}^{d}$ pour $d \geq 3$.

\section{References}

[1] T.J.BAKER; "Automatic mesch generation for complex three-dimensional regions using a constrained Delaunay triangulation", Eng. with Computers, 5, pp. 161-175, (1989).

[2] J-D.Boissonnat; "Shape reconstruction from planar cross sections", Comput. Vision Graphics Image Process., 44, pp. 1-29, (1988).

[3] J-D.Boissonnat et M.Teillaud; "A hierarchical representation of objects: The Delaunay Tree", Second ACM Symp. on Comput. Geom. in Yorktown Heights, (1986).

[4] J-D.Boissonnat, O.Devillers, R.Schott, M.Teillaud et M.Yvinec; "Applications of Random Sampling to On-Line Algorithms in Computational Geometry", Discrete Comput. Geom., 8, pp. 51-71, (1992).

[5] H.Boroucha Ki; "Triangulation de Delaunay sous contraintes dans $\mathbb{R}^{2}$ ", C. R. Acad. Sci. Paris, t. 318, Série I, pp. 367-372, (1994).

[6] H.BorouchaKi, F.Norguet ET F.SChmitT; "Algorithmes généraux d'enveloppe convexe en dimension quelconque et randomisation", Prépub. Maths. de l'U.R.A.212, Univ. Paris 7 CNRS-U.R.A.212, 50, (1992).

[7] H.Borouchaki, F.Norguet et F.SChmitt; "Graphe de connexion et triangulation dans $\mathbb{R}^{d} "$, C. R. Acad. Sci. Paris, t. 318, Série I, pp. 283-288, (1994).

[8] A.Bowyer; "Computing Dirichlet tessellations", Comput. J., 24, pp. 162-166, (1981).

[9] E.Brière de L'Isle et P-L.George; "Optimisation de maillages tridimensionnels", Rapport de recherche INRIA, no 2046, (1993).

[10] B. Chazelle; "An optimal convex hull algorithm in any fixed dimension", Discrete Comput. Geom., 10, pp. 377-409, (1993).

[11] B.Chazelle et L.Palios; "Triangulating a nonconvex polytope", Discrete Comput. Geom., 5, pp. 505-526, (1990).

[12] L-P.CHEw; "Constrained Delaunay triangulation", Proceedings, Third ACM Symposium on Computional Geometry, Waterloo, pp. 216-222, (1987). 
[13] K.L.Clarkson et P.W.Shor; "Applications of Random Sampling in Computational Geometry, II", Discrete Comput. Geom., 4, pp. 387-421, (1989).

[14] A.K.Cline et R.J.Renka; "A constrained two-dimensional triangulation and the solution of closest node problemes in the presence of barriers", SIAM J. Numer. Anl., 27(5), pp. 1305$1321,(1990)$.

[15] B.DelaunaY; "Sur la sphère vide", Izv. Akad. Nauk SSSR Otdelenie Matemat. Estestvennyka Nauk, 7, pp. 793-800, (1934).

[16] P.A.Devijver et M.Dekesel; "Computing multidimensional Delaunay tessellation", Pat. Recogn.'lett., 1, pp. 311-316, (1983).

[17] H.Edelsbrunner et N.R.Shah; "Incremental topological flipping works for regular triangulations", Proc. 8th ACM Ann. Comput. Geom. June, Berlin, Germany, pp. 43-52, (1992).

[18] L.De Floriani et E.Puppo; "An On-Line Algorithm for Constrained Delaunay Triangulation", Graphical Models and Image Process., 54(3), pp. 290-300, (1992).

[19] P-L.George; "Génération de maillages par une méthode de type Voronoi", INRIA Rapport de recherche, 1398, (1991).

[20] P-L.George, F.Hecht ET E.Saltel; "Fully automatic mesh generator for 3D domains of any shape", Impact of Comput. in Sci. and Eng., 2, pp. 187-218, (1990).

[21] L.J.Guibas ET J.STOLFI; "Primitives for the manipulation of general subdivisions and the computation of Voronoi diagram", Proc. 15th ACM Conf. on the Theory of Comput., pp. 221-234, (1983).

[22] C.HAzLewood; "Approximating constrained tetrahedrizations", Comput. Aided Geom. Design, 10, pp. 67-87, (1993).

[23] F.Hermeline; "Triangulation automatique d'un polyèdre en dimension $N$ ", RAIRO Analyse Num., 16(3), pp. 211-242, (1982).

[24] B.JoE; "Construction of three-dimensional Delaunay triangulations using local transformations", Comput. Aided Geom. Design, 8, pp. 123-142, (1991).

[25] B.Joe ET R.B.Simpson; "Triangular meshes for regions of complicated shape", Int. J. Numer. Methods Eng., 23, pp. 751-778, (1986).

[26] C.L.Lawson; "Properties of n-dimensional triangulations", Comput. Aided Geom. Design, 3, pp. 231-246, (1986).

[27] D.T.Lee ET A.K.Lin; "Generalized Delaunay Triangulation for Planar Graphs", Discrete Comput. Geom., 1, pp. 201-217, (1988). 
[28] S.Mitchell et S.Vavasis; "Quality mesh generation in three dimensions", Proc. 8th ACM Ann. Comput. Geom. June, Berlin, Germany, pp. 212-221, (1992).

[29] A.Perronnet; "A generator of tetrahedral finite elements for multi-material object and fluids", Num. grid gene. in comput. fluid mechanics'88, Miami, pp. 719-728, (1988).

[30] F.P.Preparata ET M.I.Shamos; "Computational Geometry, An Introduction", Springer-Verlag, New York, 1985.

[31] V.T.RAJan; "Optimality of the Delaunay triangulation in $\mathbb{R}^{d}$ ", Proc. 7th $A C M$ Ann. Comput. Geom., pp. 357-363, (1991).

[32] J.RUPPERT ET R. SEIDEL; "On the difficulty of triangulating three-dimensional nonconvex polyhedra", Discrete Comput. Geom., 7, pp. 227-253, (1992).

[33] N.SAPIDIS ET R.PERUCCHIO; "Delaunay triangulation of arbitrarily shaped planar domains", Comput. Aided Geom. Design, 8, pp. 421-437, (1991).

[34] R.SEIDEL; "Small-dimensional linear programming and convex hulls made easy", Discrete Comput. Geom., 6, pp. 423-434, (1991).

[35] G.Voronoï; "Nouvelles application des paramètres continus à la théorie des formes quadratiques. Deuxième mémoire: Recherches sur les paralléloèdres primitifs", J. Angew. Math., 134, pp. 167-171, (1908).

[36] D.F.Watson; "Computing the n-dimensional Delaunay tessellation with application to Voronoi polytopes", Comput.J., 24, pp. 167-172, (1981). 


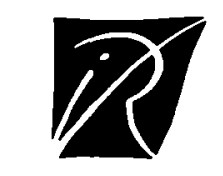

Unité de recherche INRIA Rocquencourt

Domaine de Voluceau - Rocquencourt - B.P. 105 - 78153 Le Chesnay Cedex (France)

Unité de recherche INRIA Lorraine - Technopóle de Nancy-Brabois - Campus scientifique 615, rue du Jardin Botanique - B.P. $101-54602$ Villers les Nincy Cedex (France)

Unite de recherche INRIA Rennes - IRISA. Campus universitaire de Beaulieu 35042 Rennes Cedex (France)

Unite de recherche INRIA Rhone-Alpes 46. aventue Félix Viallet - 3803 I Grenoble Cedex I (France)

Unité de recherche INRIA Sophia Antipolis - 2004, route des I ucioles - B.P. 93 - 06902 Sophia Antipolis Cedex (France)

Éditcur

INRIA - Domaine de Voluceau - Rocquencourt - B.P. 105 - 78153 Le Chesnay Cedex (France)

ISSN $0249-6399$ 